\title{
LOS PROCESOS ELECTORALES CENTROAMERICANOS EN LA DECADA DE LOS 80
}

\author{
Fernando Harto de Vera $(*)$
}

Centroamérica inicia la década de los noventa con un panorama en el que confluyen continuidades y rupturas con el pasado inmediato representado por la década anterior. En efecto, los años ochenta se inauguraron con el estallido de las contradicciones que a lo largo de los 30 años que se extienden entre la posguerra mundial y esta última fecha, presidieron el devenir histórico de las formaciones sociales del Istmo. En un proceso suficientemente conocido, esta acumulación de contradicciones configuraron la crisis orgánica que la región vivió a partir de julio de 1979. A partir de entonces, la crisis evolucionó por distintas fases hasta que finalmente se llegó a la década de los noventa con un escenario que difiere del que existía a comienzos de los ochenta. Si fuera posible reducir toda la compleja dinámica de los acontecimientos que vivió el Istmo en los ochenta a su rasgo más sobresaliente, este podría ser el del predominio de la guerra sobre la política. Sin embargo, a partir de la firma de los acuerdos de Esquipulas de agosto de 1987 , se asiste a la progresiva pero inexorable apertura de un espacio para la política que termina por imponerse a la guerra. Afirmábamos al comienzo de este párrafo que la década de los noventa dibujaba un escenario para la región centroamericana con continuidades y rupturas. Pues bien, las continuidades tienen que ver con la existencia de problemas no resueltos de índole estructural y las rupturas con los métodos que se ensayan para la resolución de los mismos: en los ochenta la guerra, en los noventa la política.

Lo anterior no significa que la política estuviera ausente de la región en los ochenta. Sin duda que existió, pero como un espacio subordinado en su dinámica y objetivos al espacio hegemónico, esto es, a la guerra. Por tanto, la primera reflexión que conviene no perder de vista es que las elecciones celebradas en década de los ochenta en Centroamérica, en tanto que procesos que pertenecen al espacio de lo político, tuvieron un valor relativo y que en cualquier caso se subordinó a la lógica de la guerra. Es decir que, con la excepción de Costa Rica, la correlación de fuerzas y la fuente de extracción de recursos de poder, no pasaba más que de modo parcial y subsidiariamente a través de los eventos electorales. Cabe entonces formularse la pregunta de si tiene sentido convertir en objeto de reflexión y análisis un fenómeno que, de acuerdo a lo que venimos argumentando, no constituye un factor explicativo de primer orden. La respuesta al mismo nos la da el cambio tendencial hacia el predominio de lo político con el que se abre la década de los noventa. No parece demasiado descabellado suponer que esta afirmación de lo político va a profundizarse en el futuro. En este nuevo contexto, las elecciones emergen como procesos que ahora comirenzan a permitir comprender y explicar una mayor parcela de la realidad política, social y económica. En este sentido, los procesos electorales de los ochenta constituyen el antecedente sobre el que las distintas fuerzas políticas deberán construir sus estrategias de actuación para el futuro.

Teniendo en cuenta las premisas anteriores, el objetivo que pretendemos con el presente artículo es el de ofrecer un cuadro que resuma cuales han sido las tendencias principales que los comicios de los ochenta han dejado en los distintos sistemas políticos del Istmo.

\section{COSTA RICA}

Constituye un tópico de obligada referencia comenzar el análisis de la evolución electoral de Costa Rica en la década de los ochenta señalando la solidez y el alto grado de consolidación que las elecciones tienen dentro de la cultura política de este país. Desde el año 1948 se suceden sin excepción y con la regularidad cuatrienal, marcada por la legislación vigente, los sucesivos procesos electorales. Dentro de esta secuencia se inscriben las elecciones presidenciales y legislativas celebradas en los años 1982, 1986 y 1990.

En las primeras elecciones de la década, celebradas en mayo de 1982, los dos fuerzas mayoritarias presentaron como candidatos a la presidencia de la República a Luis A. Monge por el Partido Liberación Nacional y a Rafael Angel Calderón Fournier por Unidad, coalición compuesta por los partidos Republicano Calderonista, Unión Popular, Demócrata Cristiano y Renovación Democrática. El triunfo en esta ocasión fue para el candidato liberacionista que consiguió alzarse con el $58.8 \%$ de los sufragios frente al $33.6 \%$ obtenido por su oponente. La izquierda que se presentó bajo la siglas de Pueblo Unido, consiguió un 3,3\% de los votos emitidos. La participación del electorado arrojó una tasa de abstencionismo del $21.4 \%$. La traducción de estos resultados porcentuales en la composición del parlamento fue la siguiente: PLN 33 escaños, Unidad 18 escaños, Pueblo Unido 4 y Otros partidos 1.

Tras estas elecciones el espectro político de la oposición al PLN, esto es la izquierda representada por Pueblo Unido y la derecha representada por Unidad, experimentaron dos tendencias diametralmente divergentes: centrífuga y centrípeta respectivamente. Así, la izquierda costarricense vivió 
a fines de 1983 un proceso de división. El Partido Comunista se escindió en dos organizaciones: Vanguardia Popular dirigida por Humberto Vargas y Arnoldo Ferreto y el Partido del Pueblo Costarricense dirigido por el líder tradicional Manuel Mora Valverde. Esta situación de división de la izquierda perduró hasta las elecciones de 1986 en la que se presentaron cada una de las dos organizaciones por separado. Por el contrario y en el mismo año de 1983, los distintos grupos de la derecha antiliberacionista confluyeron en un proceso de convergencia que llevó a la formación del Partido de Unidad Socialcristiana. A partir de esta fecha, la derecha estuvo representada en los comicios de 1986 y 1990 por esta organización.

Las elecciones presidenciales de febrero de 1986 por lo que se refiere a los dos partidos mayoritarios enfrentaron a los candidatos Oscar Arias por Liberación Nacional mientras que la oposición volvió a repetir la fórmula de los comicios anteriores al presentar a Rafael Angel Calderón esta vez bajo una nueva denominación, el Partido de Unidad Socialcristiana. Los resultados consolidaron otros cuatro años de hegemonía liberacionista al triunfar Oscar Arias con el $52.3 \%$ de los votos frente al $45.8 \%$ obtenido por el candidato socialcristiano. La izquierda se presentó a las elecciones bajo dos bloques: Alianza Popular que consiguió un $0.8 \%$ de los sufragios y Pueblo Unido que obtuvo un $0.6 \%$ de los votos. En cuanto a las legislativas el reparto de escaños fue de 29 para el PLN, 25 para el PUSC y 1 para cada organización de la izquierda. El nivel de abstencionismo se situó en el 18,2\% ligeramente por debajo del habido en los anteriores comicios.

La última convocatoria a las urnas hasta la fecha, acaecida en febrero de 1990, se sitúa a caballo de la década anterior y comienzos de la presente. De nuevo la participación del electorado fue alta con una tasa de abstencionismo del $18 \%$ El PLN presentó como candidato presidencial a Carlos Manuel Castillo mientras que el PUSC repitió por tercera vez consecutiva la misma fórmula electoral al presentar como candidato a Rafael Angel Calderón. Tras dos administraciones liberacionistas el electorado costarricense otorgó en esta ocasión su confianza al candidato socialcristiano quién se alzó con el $51.4 \%$ de los sufragios frente al 47.2\% obtenido por Carlos Manuel Castillo. Respecto a las elecciones a la Asamblea Legislativa, el PUSC obtuvo 29 escaños, lo que le dio la mayoría absoluta. El PLN obtuvo 25 escaños y el partido Pueblo Unido uno.

Los tres procesos electorales que venimos analizando muestran que la década de los ochenta ha confirmado las tendencias que desde 1948, fecha fundacional del vigente régimen político, forman parte de la democracia costarricense. Así, el sistema de partidos puede catalogarse como de bipartidismo perfecto o extremado puesto que las dos fuerzas mayoritarias aglutinan entre ambas a más del $90 \%$ de las preferencias del electorado. La crisis vivida por la izquierda que a lo largo de la década ha experimentado un serio retroceso electoral no ha hecho sino reforzar aún más esta tendencia. Dentro de esta situación de bipartidismo destaca como partido más fuerte el PLN como lo demuestra la victoria consecutiva en las elecciones de 1982 y 1986. Por otra parte, mientras que el PLN data como partido desde la década de los años cincuenta, la oposición antiliberacionista como hemos visto más arriba no logra su unificación hasta la fecha reciente de 1983. Puede por tanto afirmarse que el eje de la vida política pasa por el PLN puesto que el apoyo que logra concitar el PUSC se define más en función del antiliberacionismo que de un proyecto político delineado que trascienda la personalidad de un líder concreto.

\section{EL SALVADOR}

Si la cuestión de la democracia fuera tan simple como contabilizar el número y la periodicidad con la que se celebran elecciones, tendríamos que concluir con la boutade de que, durante la década de los ochenta, El Salvador se constituyó como el país más democrático de Centroamérica. En efecto, no hay otro país en el Istmo que pueda arrojar el siguiente balance: una elección a Asamblea Nacional Constituyente (1982), dos elecciones presidenciales (1984 y 1989) y tres elecciones legislativas y municipales (1985, 1988 y 1991).

Las elecciones de marzo de 1982 significaron el comienzo del intento de institucionalización del proceso político que se había abierto en El Salvador con posterioridad al golpe de estado de octubre de 1979. Los resultados reflejaron una composición de la Asamblea Nacional que no se ajustaba a lo previsto por los Estados Unidos. En efecto, el PDC con el $40.3 \%$ de los votos válidos se alzó con 24 escaños, ARENA con el 29.3\%, obtuvo 19 escaños y el PCN con el 19\% consiguió 14 escaños. Por lo tanto, una coalición entre ARENA y el PCN hubiera obtenido el control del Legislativo. Sin embargo, las presiones norteamericanas consiguieron imponer como presidente provisional al independiente Alvaro Magaña.

Las elecciones presidenciales de 1984 tuvieron que ser celebradas en dos vueltas al no lograr ninguno de los candidatos el número mínimo de sufragios exigidos por la ley para ser proclamado presidente de la República en la primera vuelta. Los resultados de la primera vuelta de estos comicios fueron del $43.4 \%$ para el PDC, el $29.8 \%$ para ARENA y el $19.3 \%$ para el PCN. En la segunda vuelta el triunfo fue para el candidato del PDC Napoleón Duarte quien obtuvo el $53,6 \%$ de los votos válidos frente al mayor D'Abuisson, candidato de ARENA, que obtuvo el $46.4 \%$ de los sufragios válidos. Se creó por tanto una situación de inestabilidad al estar la Presidencia de la República en manos del PDC y el legislativo en manos de ARENA. Esta situación se mantendría hasta el proceso electoral de marzo de 1985. En esa ocasión se celebraron elecciones a Diputados y Alcaldes con los siguientes resultados: PDC un $52.35 \%$ y 33 escaños, ARENA un $29.7 \%$ y 13 escaños y el PCN un $8.36 \%$ y 12 escaños. Estos resultados suponen que el PDC se alzó con el triunfo obteniendo la mayoría absoluta y por tanto encontrándose en una situación cómoda para llevar a cabo su programa electoral al dominar tanto la Presidencia de la República como el legislativo. Esta situación se mantendría hasta los comicios de 1988. Los resultados de estas elecciones centraron el interés en la modificación que se dió en la correlación de fuerzas entre el PDC y ARENA:en los porcentajes pasaron del 52.35 al $35.1 \%$ el PDC y ARENA del $29.7 \%$ al $48.1 \%$. El PCN prácticamente repitió los resultados de la anterior votación.

El periodo que va desde marzo de 1988 hasta las elec- 
ciones presidenciales de 1989 se asemeja a la situación vivida entre 1984 y 1985 sólo que en esta ocasión es al revés: será ARENA quien domine la Asamblea Legislativa y el PDC quien domine la presidencia de la república. A esta situación se le pone término con las elecciones presidenciales de 1989 en las que ARENA refrendó su triunfo del año anterior con los siguientes resultados: ARENA $53.8 \%$ de los votos válidos, PDC $36.0 \%$, PCN $4.1 \%, \mathrm{CD}$ $3.8 \%$. Un hecho nuevo de estas elecciones es que por primera vez participa la izquierda bajo las siglas de la Convergencia Democrática.

El último proceso electoral celebrado en marzo de 1991, el sexto desde la apertura del proceso de transición ocurrido con las elecciones de 1982, convocó al electorado salvadoreño para elegir diputados, alcaldes así como los representantes de este país ante el Parlamento Centroamericano. Junto con la convocatoria por vez primera a la elección al parlamento Centroamericano, este último proceso electoral se destaca de los anteriormente celebrados por varias novedades. En primer lugar, se celebró bajo la reforma del Codigo Electoral que, entre otros elementos, incluyó como principal aporte la ampliación en el número de los componentes de la Asamblea Nacional que pasó de estar integrada por 60 diputados a 84 . Los 24 nuevos escaños se repartieron de la siguiente forma: 20 escaños correspondieron a una circunscripción de carácter nacional, y dos circunscripciones, la de San Salvador y la correspondiente al Departamento de La Libertad aumentaron su dotación de representantes en 3 y 1 respectivamente. Los 20 diputados correspondientes a la circunscripción nacional, serán los encargados de representar a El Salvador en el futuro Parlamento Centroamericano, siendo sustituidos cuando tal institución comience a ser operativa, por los diputados suplentes en la Asamblea Legislativa salvadoreña.

Para comenzar el análisis de los resultados electorales por los datos referidos a la participación, hay que señalar que el total de votantes inscritos esta vez ascendió a 2.500.000 ciudadanos de los que al menos 2.150 .000 estaban en posesión de su carnet electoral. La cantidad de electores que acudieron a las urnas a depositar su voto fue de 1.138.581 por lo tanto hubo 1.442.419 abstenciones, situándose la tasa de abstención en un $44.1 \%$.

En cuanto al reparto de escaños y votos entre los diferentes partidos el balance fue como sigue: ARENA 44.33\% (39), PDC $27.96 \%$ (26), PCN $8.99 \%$ (9), MAC $3.23 \%$ (1), CD $12.16 \%$ (8), UDN 2,68\% (1).

Los resultados no significaron ninguna sorpresa y más bien tendieron a confirmar los sondeos preelectorales. En esta ocasión, la izquierda se presentó a las elecciones bajo dos formaciones, la Convergencia Democrática, que experimentaba tras las presidenciales de 1989 su segunda cita electoral y la Unión Democrática Nacionalista, coalición que incluía al Partido Comunista que concurría por vez primera a una cita electoral puesto que estuvo ausente a lo largo de la década de los ochenta. La combinación de la ampliación del número de escaños y del sistema de representación proporcional no favoreció a la izquierda, en su calidad de fuerzas minoritarias, como a nivel teórico cabía esperar. Incluso la CD a pesar de sobrepasar en número de votos al PCN obtuvo un escaño menos que esta fuerza como consecuencia del sistema de residuos y cociente electoral.

Los seis procesos electorales que se han sucedido a lo largo de la década de los ochenta y principios de los noventa trazan una imagen cuyo rasgo más sobresaliente quizás sea la elevada tasa de abstencionismo. Con excepción de las elecciones de 1982, sin duda las que contaron con un menor grado de pureza de todas las celebradas, el porcentaje del electorado que no ha ejercido su derecho al voto se sitúa como media en torno al $45 \%$. Situación que encuentra su explicación indudablemente en la situación de guerra civil en la que se vio inmerso el país durante estos años. Pero no en el sentido de que el boicot a las elecciones decretado por el FMLN haya sido la razón fundamental tal y como pusieron de manifiesto las últimas elecciones en las que el FMLN llamó a la participación sin que ello disminuyera la tasa de abstencionismo. Más bien se debe a que buena parte de la población percibió y aún sigue percibiendo que las elecciones tienen una escasa capacidad de incidencia en la realidad y que los factores de poder se estarían disputando en otro terreno diferente al del espacio electoral. Por otra parte, esta elevada tasa de abstención hace prácticamente imposible tratar de establecer alguna previsión sobre las tendencias futuras puesto que cuando ese $45 \%$ se decida a depositar su voto puede hacer saltar por los aires el sistema de partidos tal y como se ha venido configurando. Esta configuración ha sido la de un sistema pluripartidista en el que la diferente aceptación de cada partido por el electorado ha atravesado por distintos momentos. Así, el PDC vivió entre los comicios de 1982 y los de 1985 un proceso de alza que los elevó desde el $40 \%$ hasta el $52 \%$. Este fue el momento de mayor auge de los democratacristianos puesto que a partir de este momento experimenta un proceso de caída que lo lleva a perder votos elección tras elección hasta situarse con el $28 \%$ en las últimas. Por lo que se refiere al actual partido gobernante ARENA, durante los tres primeros comicios se mantiene estable en torno al $29 \%$. Su alza es paralela a la tendencia a la baja del PDC, situándose en las elecciones de 1988 en el $48 \%$ y llegando al máximo obtenido en los comicios de 1989 con el $53.8 \%$. Este parece ser su techo electoral puesto que en las últimas elecciones ha descendido por debajo de lo obtenido en 1988 consiguiendo un 44\%. La posición de tercer partido la ostentó hasta los últimos comicios el PCN si bien con una tendencia a la baja que le hizo evolucionar desde el $19 \%$ en las dos primeras elecciones hasta el $4.1 \%$ en las del 89 . Tendencia a la baja que se rompe en las últimas elecciones en las que consigue casi el $9 \%$. Por último, la izquierda representada por la CD participa por primera vez en unas elecciones en las del año 1989 obteniendo un exiguo $3.8 \%$, resultado que remontó espectacularmente en la de 1991 en las que se alzó con el $12.1 \%$, logrando así la condición de tercera fuerza política. Por último, el resto de las formaciones minoritarias han mostrado una tendencia a la disminución de su caudal de votos lo que es interpretable como un indicador de la polarización de la sociedad salvadoreña.

\section{GUATEMALA}

El proceso de transición política en Guatemala se inaugura con las elecciones a Asamblea Nacional constituyente 
de 1984. La continuidad del proceso se completó hasta la fecha con la celebración de dos comicios que tuvieron lugar en 1985 y 1990.

En el mes de julio de 1984 se celebraron las elecciones a Asamblea Nacional Constituyente. Los resultados electorales arrojaron una composición de la cámara con una alta fragmentación de la representación. Si agrupamos estos resultados por bloques obtenemos que los partidos tradicionales (DGC, MLN-CAN, PR, PNR, PID, FUR y FUN) lograron en conjunto alzarse con el $52,5 \%$ de los sufragios lo que supuso el dominio de la Asamblea al traducirse en el control del 74\% (65 escaños) de los escaños disponibles. En segundo lugar se situó la opción de centro de reciente formación y representada por la UCN quién logró el 13,7\% de los sufragios emitidos y un 24\% (21 escaños) de los escaños en juego. Por último los partidos nuevos obtuvieron el favor de un $10,8 \%$ de los votos emitidos lo que significó un $2 \%$ (dos escaños) de los escaños.

El proceso de transición tuvo su continuidad con la celebración de elecciones presidenciales en 1985. La primera vuelta tuvo lugar en noviembre de 1985 y arrojó los siguientes resultados: en primer lugar la Democracia Cristiana se alzó con el $34,25 \%$ de los votos emitidos. En segundo lugar estuvo la UCN que obtuvo el $17,49 \%$ de los votos emitidos. Por tanto estas fueron las dos fuerzas que pasaron a disputar la segunda vuelta. El resto de los partidos y coaliciones obtuvo en esta primera vuelta los siguientes resultados: PDCN-PR obtuvo el $12,19 \%$, PSD obtuvo el $2,74 \%$. Esta primera vuelta mostró el rechazo de la población a las opciones tradicionales de derecha como el MLN, el PR y aquellos partidos de clara vinculación con los militares o que habían participado con ellos como el PID, el CAN y el PR. En la segunda vuelta, celebrada el 8 de Diciembre, el Candidato de la DC Vinicio Cerezo, obtuvo el $68.37 \%$ del total de los votos emitidos frente al $31.6 \%$ obtenido por Jorge Carpio, candidato de la UCN. Por lo que se refiere a la abstención, hay que anotar un elevado índice de participación que en su punto más bajo, la segunda vuelta, se situó en el $65 \%$ del electorado inscrito.

En junio de 1990 se convocó a un nuevo proceso electoral durante el cual se procedió a elegir al Presidente y Vicepresidente de la República, Diputados al congreso de la república, diputados para el parlamento centroamericano y alcaldes y concejales de las corporaciones. La participación en estas elecciones fue menor que en los comicios de 1985: mientras que en aquella ocasión el porcentaje de abstención rondó el $39 \%$, en estos últimos comicios aumentó hasta el 52\%. La primera vuelta tuvo lugar durante el mes de noviembre de 1990, proclamándose ganador el candidato de la UCN Jorge Carpio con el $25.7 \%$, seguido por Jorge Serrano candidato del MAS que obtuvo el $24,1 \%$; el tercer lugar correspondió al partido de gobierno DGC (17,5\%), seguido muy de cerca por el PAN $(17,3 \%)$. Los demás partidos se situaron por debajo del $5 \%$ de las preferencias del electorado. La segunda vuelta se celebró en el mes de enero de 1991 entre los candidatos del MAS y de la UCN. Las urnas dieron la victoria a Jorge Serrano con un $68.1 \%$ frente al $31.9 \%$ de su rival. Si tenemos en cuenta los porcentajes obtenidos por ambos candidatos en la primera vuelta, es evidente que el triunfo electoral de Jorge Serrano se debió a la agrupación del electorado conservador que se reveló más numeroso que el electorado centrista. Por lo que respecta a la composición del Congreso, el reparto de escaños quedó como sigue: UCN 41, DGC 27, MAS 18, PAN 12, MLN 4, Otros 14.

La característica que con más fuerza emerge de los procesos electorales analizados es sin lugar a dudas, la falta de consolidación del sistema de partidos. La extrema fragmentación de la representación en el Congreso es un claro exponente de que el sistema de partidos en Guatemala se haya aún lejos de haberse definido.

\section{HONDURAS}

En la década de los ochenta Honduras ha vivido cuatro procesos electorales. De ellos el primero (1980) revistió el carácter de elecciones a Asamblea Nacional Constituyente mientras que los siguientes (1981, 1985 y 1989) lo fueron para elegir al Presidente de la República.

Las elecciones del año 1980 fueron el acto fundacional del proceso de transición política en Honduras tras la experiencia del reformista militar que dominó la escena durante la década de los setenta. En esta ocasión, al lado de las fuerzas políticas tradicionales-Partido Liberal y Partido Nacional-se permitió la participación de nuevos actores, concretamente, el Partido de Innovación y Unidad (PINU). Sin embargo, los resultados electorales favorecieron abrumadoramente a los partidos tradicionales quienes se repartieron conjuntamente más del $90 \%$ de los sufragios del electorado. El triunfo correspondió al Partido Liberal quien se alzó con el $51 \%$ de los votos lo que significó un rechazo del electorado al Partido Nacional tradicional aliado de los militares.

Una vez concluida la redacción de la constitución, el proceso de transición se continúa con la celebración de elecciones presidenciales en noviembre de 1981. La apertura política que había supuesto la ampliación del juego partidario con la legalización y participación del PINU, se amplía en esta ocasión al permitirse la participación de la Democracia Cristiana. Sin embargo este proceso de apertura es tímido como lo expresa el hecho de que los partidos de izquierda no fueron permitidos y se vieron obligados a participar en estas elecciones bajo la fórmula electoral de candidatos independientes. Los resultados confirmaron las tendencia del anterior evento electoral. Así, por un lado, los partidos tradicionales volvieron a obtener más del $90 \%$ de los votos. Por otro, de nuevo fue el partido liberal la fuerza política que se alzó con el triunfo, convirtiéndose su candidato Roberto Suazo Córdoba, en el Presidente de la República.

Las elecciones de 1985 registraron como principal novedad respecto a los anteriores comicios, la eclosión de corrientes internas en el seno de las dos fuerzas políticas mayoritarias. Por lo que se refiere al gobernante Partido Liberal, el fenómeno tiene que ver con el estilo de la gestión de Suazo Córdova, caracterizada por el abandono del programa renovador y su progresiva derechización así como por la obsesión por nombrar como su sucesor a un candidato que respondiera a sus dictados. El resultado fue la fragmentación del Partido Liberal en distintas plataformas agrupadas en torno a candidatos que representaban a aquellos sectores del Partido opuestos a los métodos del 
presidente saliente. De este modo, bajo el marchamo del Partido liberal, se presentaron a las elecciones las siguientes corrientes y candidatos: Alianza Movimiento Liberal Azconista-ALIPO (José Azcona), Movimiento Liberal Rodista (Oscar Mejía), Movimiento Rodista (Efraín Bú) y Movimiento Liberal Democrático Revolucionario (Carlos Reina).

Por lo que respecta al Partido Nacional, el factor que explica su fragmentación en distintas corrientes internas tiene que ver con la puesta en marcha de un proceso de renovación provocado ante la coyuntura de la pérdida de las elecciones de 1980 y 1981. Tres fueron las plataformas electorales con las que el Partido Nacional concurrió a las elecciones de 1985: Movimiento Nacionalista Rafael Leonardo Callejas (Rafael Callejas), Movimiento de Cambio y Unidad (Juan Pablo Urrutia) y Movimiento Pro Candidatura de Fernando Lardizábal (Fernando Lardizábal).

Para completar este proceso electoral "a la uruguaya" durante el primer semestre de 1985 se aprobó una reforma al sistema electoral mediante la cual se introdujo un sistema muy similar a la ley de lemas de Uruguay por el que los votos de cada uno de los candidatos presentado por cada partido se sumaban al candidato del mismo con mayor número de votos. El resultado fue que José Azcona se convirtió en presidente electo gracias a la suma de los votos obtenidos por todos los candidatos del Partido Liberal (787.953 o sea un poco más del 50\%). Sin embargo, hay que tener en cuenta que el mayor número de votos obtenido individualmente por un candidato correspondió al candidato del partido Nacional Rafael Leonardo Callejas (640.000 votos) cifra superior a la votación obtenida por el expresidente Suazo Córdova. La fragmentación del voto en estas elecciones produjo una situación de elecciones sin ganador que dio como consecuencia un proceso de intensas negociaciones tanto inter como intrapartidarias. Se concretó así un pacto entre Callejistas y Azconistas que consistió en un reparto de cargos públicos y posiciones de poder en el seno de los diferentes niveles de la Administración pública Hondureña. El Partido Nacional salió claramente favorecido de este reparto como lo demuestra la preeminencia que alcanzó en el Tribunal Nacional de Elecciones, organismo clave desde el que el Partido Nacional abordaría con una clara posición ventajosa los futuros comicios. Estos se celebraron en noviembre de 1989, dando el triunfo al candidato del partido nacional, Rafael Leonardo Callejas con el $52 \%$ de los votos. Se confirmó así la tendencia presente en los anteriores comicios por la que el desgaste del partido Liberal vencedor en los procesos electorales de 1980, 1981 y 1985 iba siendo capitalizado por el partido Nacional.

En síntesis, los cuatro procesos electorales que han tenido lugar a lo largo de la década de los ochenta han configurado las siguientes tendencias:

a) Un sistema político bipartidista en el que las dos fuerzas mayoritarias el PN y el PL aglutinan en conjunto más del $90 \%$ de los sufragios emitidos. El resto de los partidos, el PDC y el PINU experimentan una tendencia al estancamiento sin que sean capaces de romper la tendencia al bipartidismo. b) Una elevada tasa de participación del electorado puesto que la tasa de abstención se mantiene en torno al $20 \%$.

\section{NICARAGUA}

En los diez años durante los cuales el sandinismo estuvo en el poder tuvieron lugar dos procesos electorales (1984 y 1990) con un significado, alcance y consecuencias radicalmente diferentes.

Los primeros comicios, celebrados en noviembre de 1984, tuvieron como objetivo la institucionalización del proceso revolucionario así como el refrendo electoral de la legitimidad que el Frente Sandinista derivaba de su exitosa intervención en la insurrección popular que acabó con la dictadura de Somoza. Asimismo y en un contexto definido por el acoso que el régimen sufría por la guerra de agresión financiada e impulsada por los Estados Unidos el valor instrumental y simbólico de estos comicios también tenía por objeto poner de manifiesto la ilegitimidad de la estrategia norteamericana para derribar a un gobierno democráticamente elegido por los votantes. Todas estas consideraciones, unida a la presión que el gobierno sandinista recibió de alguno de sus aliados motivó que se adelantará la fecha de celebración de las elecciones programada originalmente para el año 1985. La convocatoria electoral para elegir al Presidente y Vicepresidente de la República, así como los diputados que integrarían la Asamblea Legislativa, atrajo a las urnas a 1.170.142 nicaragüenses, es decir, el 75,4\% de los votantes inscritos.

Los resultados porcentuales de las presidenciales y las legislativas fueron prácticamente idénticos: Frente Sandinista de Liberación Nacional 67\%; Partido Conservador Demócrata 14\%; Partido Liberal Institucional 9,6\%; Partido Popular Socialcristiano 5,6\%; Partido Comunista de Nicaragua 1,5\%; Partido Socialista Nicaragüense $1,3 \%$ y Movimiento de Acción Popular Marxista Leninista 1\%. Estos porcentajes se tradujeron en el triunfo de los candidatos a Presidente y Vicepresidente de la República del FSLN, Daniel Ortega y Sergio Ramírez. Por lo que se refiere al reparto de escaños en la Asamblea Nacional, ésta quedó conformada como sigue: FSLN 61 escaños; PCD 14; PLI 9; PPSC 6 y los grupos PCN, PSN y MAP-ML con dos escaños cada uno.

Estos resultados pusieron de manifiesto la fortaleza de FSLN así como su arraigo popular al contar con el apoyo de casi dos tercios del electorado en unas elecciones que según los observadores internacionales contaron con las máximas garantías de limpieza y honestidad. El único factor que empañó en alguna medida el espectacular triunfo sandinista fue la negativa del principal grupo opositor a participar en el evento. En efecto, la Coordinadora Democrática formada por las organizaciones Partido Social Cristiano, Partido Socialdemócrata y Partido Liberal Constitucionalista, liderada por Arturo Cruz, sucumbió a las presiones norteamericanas en favor de posiciones abstencionistas en un intento de minar la legitimidad de las elecciones.

Entre estos comicios de 1984 y los siguientes, celebrados en el mes de febrero de 1990 , se sitúa la dinámica de paz y concertación regional que significó la firma de los 
Acuerdos de Esquipulas en agosto de 1987. El gobierno sandinista, dando cumplimiento a uno de los aspectos centrales de los acuerdos, impulsó un proceso de diálogo y negociación con la oposición, que cuajó, entre otras medidas, con el consenso en torno a la Ley Electoral y la firma del Acuerdo Político Nacional en Agosto de 1989. En dicho documento, se fija el mes de febrero de 1990 como fecha para la celebración de elecciones en las que la oposición se compromete a participar. Por tanto, las expectativas de las distintas fuerzas políticas ante la nueva convocatoria a las urnas conferían a estos comicios un carácter distintos al de los anteriores. En efecto, para el FSLN, estas elecciones suponían la última oportunidad para acabar con la guerra y tratar de conquistar la legitimidad internacional que las elecciones de 1984 no fueron capaz de proporcionarles. Para la oposición, su compromiso de participación, suponía la aceptación explícita de las reglas del juego de la institucionalidad de la revolución, algo que hasta entonces se había resistido a admitir. Además, constituía una apuesta que en caso de perderla la condenaría a tener que admitir su equivocación al cuestionar a lo largo de estos diez años la legitimidad de la hegemonía popular del FSLN.

La jornada electoral que tuvo lugar el 25 de febrero de 1990 supuso la renovación total en todos los niveles del sistema político. En ese día se celebraron elecciones a la Presidencia y Vicepresidencia de la República, a la Asamblea Nacional. a los Municipios y a los Consejos Regionales de las regiones autónomas del Atlántico norte y del Atlántico Sur.

La mayoría de los grupos políticos opositores se agruparon en la coalición Unión Nacional Opositora integrada por 14 partidos. Fuera de la UNO se presentaron grupos muy minoritarios de los que cabe destacar al Movimiento de Unidad Revolucionaria y al Partido Socialcristiano.

De la importancia que la población atribuyó a estos comicios da idea las altas cifras de participación electoral. Emitieron su voto el $86.2 \%$ de los ciudadanos inscritos.

Los resultados electorales constituyeron una sorpresa que contradijo la inmensa mayoría de los sondeos preelectorales. En porcentaje de votos los resultados fueron los siguientes: UNO 54,7\%; FSLN 40,8\%; MUR 1,2\% y PSC $0,8 \%$. La UNO elevó como Presidenta de la República a su candidata Violeta Chamorro que derrotaba al hasta entonces presidente Daniel Ortega.

En el reparto de escaños en la Asamblea Nacional, el FSLN obtuvo 38 representantes en virtud de sus votos. A estos se le sumó un representante más, correspondiente al candidato del FSLN a la presidencia de la República, puesto que la ley establecía que aquellos candidatos a la Presidencia que no resultaron elegidos y que alcanzaran o superaran el $1 \%$ de los votos válidos estarían presentes en la Asamblea Nacional. Por tanto obtuvo un total de 39 representantes. En las mismas circunstancias incurrió el candidato a la Presidencia de la República por el MUR, obteniendo así, un representante ante la Asamblea Nacional. La Alianza compuesta por el Partido Social Cristiano y el Partido Popular Socialcristiano obtuvo un representante en la Región Autónoma del Atlántico Norte. Finalmente la UNO, coalición triunfadora de las elecciones a la Asamblea Nacional se alzó con un total de 51 representantes.

El escenario político dibujado por estos dos comicios electorales revela que en Nicaragua existe un sistema de partidos caracterizado por la presencia de un actor fundamental, el FSLN. El resto de las agrupaciones y organizaciones son extremadamente débiles en términos de su penetración en la sociedad civil nicaragüense: ni la unión de todos los partidos de oposición logra igualar los resultados del FSLN en las elecciones de 1984. Por otra parte, y pese a perder indiscutiblemente las elecciones de 1990 , el FSLN conserva un nada despreciable $40 \%$ del electorado y ello en unas condiciones que como las que presidieron el contexto en el que tuvieron lugar dichos comicios, hace plausible la hipótesis de que sea el techo mínimo por debajo del cual es difícil pensar que decaiga su arraigo entre la población.

\section{RESUMEN}

En este artículo se traza cuales han sido los resultados de los distintos procesos electorales que han tenido lugar durante la década de los ochenta en Centroamérica.

\section{BIBLIOGRAFIA}

- Alcántara Saez M. Sistemas Políticos de América Latina, Ed. Tecnos, volumen II, Madrid, 1990.

- Casaus M. y Castillo R. El inicio del proceso de apertura política en Guatemala, CEDEAL, Madrid, 1989.

- Fiallos Oyanguren M. "Las elecciones en Nicaragua” en Sistemas electorales y representación política en Latinoamerica, Fundación Fiedrich Ebert, Madrid, 1986.

- Hernández Valle R. “Costa Rica: elecciones generales de 1986” en Sistemas electorales y representación política en Latinoamerica, op. cit.

- Montes S. "Las elecciones del 20 de Marzo de 1988" en ECA, N. 473-474, Marzo-Abril 1988, UCA, S. Salvador.

- Oseguera de Ochoa M. Honduras Hoy: Sociedad y crisis política, CEDOH-CRIES, Tegucigalpa, 1987.

- Varios Autores Boletín Electoral Latinoamericano, CAPEL, S. José, (números correspondientes a cada una de las distintas elecciones).

- Centroamérica en cifras, IICA-FLACSO, S. José, 1991. 